\title{
OBSERVATIONS OF THE UNIDENTIFIED GAMMA-RAY SOURCE TeV J2032+4130 BY VERITAS
}

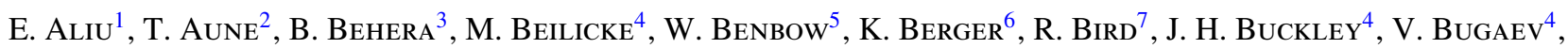
J. V. Cardenzana ${ }^{8}$, M. Cerruti ${ }^{5}$, X. Chen $^{3,9}$, L. CiupiK ${ }^{10}$, M. P. Connolly ${ }^{11}$, W. Cui ${ }^{12}$, C. Duke $^{13}$, J. Dumm $^{14}$, M. Errando ${ }^{1}$, A. Falcone ${ }^{15}$, S. Federici ${ }^{3,9}$, Q. Feng ${ }^{12}$, J. P. Finley ${ }^{12}$, P. Fortin ${ }^{5}$, L. Fortson ${ }^{14}$, A. Furniss ${ }^{16}$, N. Galante ${ }^{5}$, G. H. Gillanders ${ }^{11}$, S. Griffin ${ }^{17}$, S. T. Griffiths ${ }^{18}$, J. Grube ${ }^{10}$, G. GyuK ${ }^{10}$, D. Hanna ${ }^{17}$, J. Holder ${ }^{6}$, G. Hughes $^{3}$, T. B. Humensky ${ }^{19}$, P. KaAret ${ }^{18}$, Oleg Kargaltsev ${ }^{20}$, M. Kertzman ${ }^{21}$, Y. Khassen ${ }^{7}$, D. Kieda ${ }^{22}$, H. Krawczynski ${ }^{4}$, M. J. Lang ${ }^{11}$, A. S. Madhavan ${ }^{8}$, G. Maier ${ }^{3}$, P. Majumdar ${ }^{2,23}$, A. McCAnN ${ }^{24}$, P. Moriarty ${ }^{25}$, R. MukherJeE ${ }^{1}$, D. Nieto ${ }^{1}$, A. O’Faoláin de Bhróithe ${ }^{7}$, R. A. Ong ${ }^{2}$, A. N. Otte ${ }^{26}$, D. Pandel ${ }^{27}$, J. S. Perkins ${ }^{28}$, M. Pohl $^{3,9}$, A. Popkow $^{2}$, H. Prokoph $^{3}$, J. Quinn ${ }^{7}$, K. RagaN ${ }^{17}$, J. Rajotte ${ }^{17}$, L. C. Reyes ${ }^{29}$, P. T. ReYnolds ${ }^{30}$, G. T. Richards ${ }^{26}$, E. RoACHE ${ }^{5}$, G. H. Sembroski ${ }^{12}$, C. Skole $^{3}$, D. StaszaK ${ }^{17}$, I. Telezhinsky ${ }^{3,9}$, M. Theiling ${ }^{12}$, J. V. Tucci ${ }^{12}$, J. Tyler ${ }^{17}$, A. Varlotta ${ }^{12}$, S. Vincent $^{3}$, S. P. WaKely ${ }^{31}$, T. C. WeEkes ${ }^{5}$, A. Weinstein ${ }^{8}$, R. Welsing ${ }^{3}$, D. A. Williams ${ }^{16}$, and B. ZitZer ${ }^{32}$

${ }^{1}$ Department of Physics and Astronomy, Barnard College, Columbia University, NY 10027, USA

${ }^{2}$ Department of Physics and Astronomy, University of California, Los Angeles, CA 90095, USA; pratik.majumdar@saha.ac.in

${ }^{3}$ DESY, Platanenallee 6, D-15738 Zeuthen, Germany; gareth.hughes@ desy.de

${ }^{4}$ Department of Physics, Washington University, St. Louis, MO 63130, USA

${ }^{5}$ Fred Lawrence Whipple Observatory, Harvard-Smithsonian Center for Astrophysics, Amado, AZ 85645, USA

${ }^{6}$ Department of Physics and Astronomy and the Bartol Research Institute, University of Delaware, Newark, DE 19716, USA

${ }^{7}$ School of Physics, University College Dublin, Belfield, Dublin 4, Ireland

${ }^{8}$ Department of Physics and Astronomy, Iowa State University, Ames, IA 50011, USA

${ }^{9}$ Institute of Physics and Astronomy, University of Potsdam, D-14476 Potsdam-Golm, Germany

${ }^{10}$ Astronomy Department, Adler Planetarium and Astronomy Museum, Chicago, IL 60605, USA

${ }^{11}$ School of Physics, National University of Ireland Galway, University Road, Galway, Ireland

${ }^{12}$ Department of Physics, Purdue University, West Lafayette, IN 47907, USA

${ }^{13}$ Department of Physics, Grinnell College, Grinnell, IA 50112-1690, USA

${ }^{14}$ School of Physics and Astronomy, University of Minnesota, Minneapolis, MN 55455, USA

${ }^{15}$ Department of Astronomy and Astrophysics, 525 Davey Lab, Pennsylvania State University, University Park, PA 16802, USA

${ }^{16}$ Santa Cruz Institute for Particle Physics and Department of Physics, University of California, Santa Cruz, CA 95064, USA

${ }^{17}$ Physics Department, McGill University, Montreal, QC H3A 2T8, Canada

${ }^{18}$ Department of Physics and Astronomy, University of Iowa, Van Allen Hall, Iowa City, IA 52242, USA

${ }^{19}$ Physics Department, Columbia University, New York, NY 10027, USA

${ }^{20}$ Department of Physics, The George Washington University, Washington, D.C. 20052 USA

${ }^{21}$ Department of Physics and Astronomy, DePauw University, Greencastle, IN 46135-0037, USA

${ }^{22}$ Department of Physics and Astronomy, University of Utah, Salt Lake City, UT 84112, USA

${ }^{23}$ Saha Institute of Nuclear Physics, Kolkata 700064, India

${ }^{24}$ Kavli Institute for Cosmological Physics, University of Chicago, Chicago, IL 60637, USA

${ }^{25}$ Department of Life and Physical Sciences, Galway-Mayo Institute of Technology, Dublin Road, Galway, Ireland

${ }^{26}$ School of Physics and Center for Relativistic Astrophysics, Georgia Institute of Technology, 837 State Street NW, Atlanta, GA 30332-0430, USA

${ }^{27}$ Department of Physics, Grand Valley State University, Allendale, MI 49401, USA

${ }^{28}$ N.A.S.A./Goddard Space-Flight Center, Code 661, Greenbelt, MD 20771, USA

${ }^{29}$ Physics Department, California Polytechnic State University, San Luis Obispo, CA 94307, USA

${ }^{30}$ Department of Applied Physics and Instrumentation, Cork Institute of Technology, Bishopstown, Cork, Ireland

${ }^{31}$ Enrico Fermi Institute, University of Chicago, Chicago, IL 60637, USA

32 Argonne National Laboratory, 9700 South Cass Avenue, Argonne, IL 60439, USA

Received 2013 September 10; accepted 2014 January 8; published 2014 February 7

\section{ABSTRACT}

TeV J2032+4130 was the first unidentified source discovered at very high energies (VHEs; $E>100 \mathrm{GeV}$ ), with no obvious counterpart in any other wavelength. It is also the first extended source to be observed in VHE gamma rays. Following its discovery, intensive observational campaigns have been carried out in all wavelengths in order to understand the nature of the object, which have met with limited success. We report here on a deep observation of TeV J2032+4130 based on $48.2 \mathrm{hr}$ of data taken from 2009 to 2012 by the Very Energetic Radiation Imaging Telescope Array System experiment. The source is detected at 8.7 standard deviations $(\sigma)$ and is found to be extended and asymmetric with a width of 9.5 \pm 1.2 along the major axis and $4^{\prime} .0 \pm 0.5$ along the minor axis. The spectrum is well described by a differential power law with an index of $2.10 \pm 0.14_{\text {stat }} \pm 0.21_{\text {sys }}$ and a normalization of $\left(9.5 \pm 1.6_{\text {stat }} \pm 2.2_{\text {sys }}\right) \times 10^{-13} \mathrm{TeV}^{-1} \mathrm{~cm}^{-2} \mathrm{~s}^{-1}$ at $1 \mathrm{TeV}$. We interpret these results in the context of multiwavelength scenarios which particularly favor the pulsar wind nebula interpretation.

Key words: gamma rays: general - pulsars: individual (PSR J2032+4127)

Online-only material: color figures

\section{INTRODUCTION}

The Cygnus $\mathrm{X}$ complex is one of the brightest areas of the sky in all wavelengths and is host to a large number of sources and source types. These include active star forming regions, pulsars, and supernova remnants (SNRs). Objects connected to this region include the star association Cygnus OB2, the microquasar Cygnus X-3, the SNR G78.2+2.1, and TeV J2032+4130 
Table 1

Summary of VHE Detections, Measured Positions, and Extensions for Each Experiment Prior to VERITAS Observations

\begin{tabular}{|c|c|c|c|c|c|}
\hline Experiment & R.A. & $\begin{array}{l}\text { Error in R.A. } \\
\text { Stat }(\text { Sys }) \\
\quad(\operatorname{arcmin})\end{array}$ & Decl. & $\begin{array}{l}\text { Error in Decl. } \\
\text { Stat }(\text { Sys }) \\
(\operatorname{arcmin})\end{array}$ & $\begin{array}{c}\text { Reported Extension } \\
\pm S t a t(S y s) \\
(\operatorname{arcmin})\end{array}$ \\
\hline HEGRA & $20^{\mathrm{h}} 31^{\mathrm{m}} 57^{\mathrm{s}}$ & $6.2(13.7)$ & $+41^{\circ} 29^{\prime} 57^{\prime \prime}$ & $1.1(1.0)$ & $6.2 \pm 1.2(0.9)$ \\
\hline Whipple & $20^{\mathrm{h}} 32^{\mathrm{m}} 27^{\mathrm{s}}$ & 21(23) & $+41^{\circ} 39^{\prime} 17^{\prime \prime}$ & $5(6)$ & $<6.0$ \\
\hline MAGIC & $20^{\mathrm{h}} 32^{\mathrm{m}} 20^{\mathrm{s}}$ & 11(11) & $+41^{\circ} 30^{\prime} 36^{\prime \prime}$ & $1.2(1.8)$ & $5.0 \pm 1.7(0.6)$ \\
\hline Milagro & $20^{\mathrm{h}} 28^{\mathrm{m}} 43^{\mathrm{s}} .2$ & 25 & $+41^{\circ} 07^{\prime} 48^{\prime \prime}$ & 16 & 66 \\
\hline ARGO & $20^{\mathrm{h}} 32^{\mathrm{m}} 24^{\mathrm{s}} .0$ & $\cdots$ & $+41^{\circ} 45^{\prime} 00^{\prime \prime}$ & $\cdots$ & $12_{-12}^{+24}$ \\
\hline
\end{tabular}

Notes. Also shown are the reported right accessions and declinations (J2000), their corresponding errors and measured extensions of the source. Systematic errors are shown in parentheses.

making it a natural laboratory for the study of cosmic ray acceleration. TeV J2032+4130 was discovered serendipitously by the HEGRA imaging atmospheric Cherenkov telescope (IACT) system (Aharonian et al. 2002, 2005) during observations made in the years 1999-2001. It was the first TeV gamma-ray detection to have no obvious counterpart at any other wavelength and was also the first extended source to be discovered in the very high energy (VHE) range. Analysis of combined HEGRA data from 1999-2002 gave a final position for the extended VHE source of R.A. $=20^{\mathrm{h}} 31^{\mathrm{m}} 57^{\mathrm{s}} .0 \pm 66^{\prime} \cdot 2_{\text {stat }} \pm 13^{\prime \prime} \cdot 7_{\text {sys }}$, decl. $=$ $+41^{\circ} 29^{\prime} 57^{\prime \prime} \pm 1^{\prime} 1_{\text {stat }} \pm 1{ }^{\prime} 0_{\text {sys }}$ (excess events center of gravity), and a Gaussian standard deviation width of $\sigma=6.2 \pm 1$ '. $2_{\text {stat }} \pm$ $0{ }^{\prime} 9_{\text {sys }}$ (Aharonian et al. 2005). HEGRA reported an integral gamma-ray flux above $1 \mathrm{TeV}$ of $(6.9 \pm 1.8) \times 10^{-13} \mathrm{~cm}^{-2} \mathrm{~s}^{-1}$ with spectral index of $1.9 \pm 0.3$.

Following the discovery, an archival search of the Whipple $10 \mathrm{~m}$ telescope data was carried out that showed evidence of a source consistent with TeV J2032+4130 (Lang et al. 2004). However, it is worth noting that the peak emission in the Whipple data had an offset of $\sim 3.6$ with respect to the HEGRA position. The gamma-ray flux measured by Whipple was $12 \%$ of the Crab nebula flux above $400 \mathrm{GeV}$. Based on observations carried out by Whipple in 2003-05, and assuming a spectral shape the same as that of the Crab nebula, it was later reported to be $8 \%$ of the Crab nebula flux (Konopelko et al. 2007). The MAGIC collaboration, too, has reported a deep exposure of this region (Albert et al. 2008). The MAGIC collaboration has also found the source to be extended, with an integral flux and spectral index comparable to that measured by HEGRA. These measurements have been extended to even higher energies by the air shower array detectors Milagro and ARGO (Abdo et al. 2012; The ARGO-YBJ Collaboration et al. 2013). Summaries of the positions and morphologies of the results discussed can be found in Table 1.

Since the discovery of TeV J2032+4130, several observations of the region have been made by X-ray telescopes including Chandra and XMM-Newton, which operate in the energy range of $0.1-10 \mathrm{keV}$ and $0.2-12 \mathrm{keV}$, respectively. Multiple ( 20) point sources were detected in a $5 \mathrm{ks}$ Chandra observation (Butt et al. 2003). A deep follow-up $50 \mathrm{ks}$ observation yielded 240 X-ray sources within the same field of view (Butt et al. 2006). A $\sim 50 \mathrm{ks} X M M-N e w t o n$ exposure was also obtained (Horns et al. 2007). After the known X-ray sources were subtracted, Horns et al. (2007) reported an extended X-ray emission region with a FWHM of $\sim 12$ arcmin. An analysis of the Chandra data also showed the presence of diffuse X-ray emission; however, low photon statistics did not allow for a detailed study of the spectrum. Mukherjee et al. (2003) carried out optical observations of several of the brightest X-ray sources and found that most of these were either $\mathrm{O}$ stars or foreground late-type stars.

Observations were also made by Suzaku (Murakami et al. 2011 ) in the energy range $2-10 \mathrm{keV}$. The authors found two structures within the $\mathrm{TeV}$ gamma-ray emission region. After estimating the contribution from the point sources identified by Chandra, the X-ray spectra of the diffuse components were extracted. The diffuse X-ray spectrum was best-fit with a powerlaw with a photon index of $\sim 2$.

Radio observations of the region have been made using the Giant Metrewave Radio Telescope (Paredes et al. 2007), yielding several radio sources. At least three of the sources were reported to be non-thermal along with an extended non-thermal diffuse emission.

A previously unknown gamma-ray pulsar, PSR J2032+4127, with a pulse period of $142 \mathrm{~ms}$, was discovered in a blind search by Fermi-Large Area Telescope (LAT), located 0.07 from the center of the HEGRA detection (Camilo et al. 2009). Subsequent radio measurements made by the Green Bank Telescope (Camilo et al. 2009) localized the position to within a few arcseconds. A characteristic age of $0.11 \mathrm{Myr}$ and a spin-down power of $2.7 \times$ $10^{35} \mathrm{erg} \mathrm{s}^{-1}$ were derived (Camilo et al. 2009). A dispersion measure of $114.8 \pm 1.0 \mathrm{pc} \mathrm{cm}^{-3}$ resulted in a distance of $3.6 \mathrm{kpc}$ when standard models for dispersion in the Milky Way were applied (Cordes \& Lazio 2002). However, based on the pulsar's gamma-ray luminosity, a revised estimate of $1.7 \mathrm{kpc}$ was suggested (Camilo et al. 2009), which would place it at the same distance as the Cygnus OB2 star-forming region. The discovery of the pulsar has led several authors to establish a connection between TeV J2032+4130 and PSR J2032+4127 (for more details see Cui 2009). This argument has been strengthened by the detection of the X-ray emission that is spatially coincident with TeV J2032+4130. However, arguments based on spatial coincidence alone can be suspect due to the fact that the morphology of the source can be different at different wavelengths. In order to understand the nature of the emission, it is important to study the morphology of the source with a detector with an improved sensitivity and angular resolution. It is clear from the above discussions that despite several attempts to unravel the nature of TeV J2032+4130 since its discovery, the source along with its position, flux and morphology remains a mystery. This prompted Very Energetic Radiation Imaging Telescope Array System (VERITAS) to perform a deep observation of this very interesting region of the Galaxy with the aim of better understanding the morphology and source position.

The paper is organized as follows. Section 2 describes the VERITAS experiment and observations made on $\mathrm{TeV}$ J2032+4130. Section 3 describes the results obtained, and 
Section 4 presents a Fermi-LAT analysis of the region of $\mathrm{TeV} \mathbf{J} 2032+4130$. Finally, we discuss the implications of our observations on the source in the context of multiwavelength observations.

\section{VERITAS OBSERVATIONS}

The VERITAS array of IACTs is located at the Fred Lawrence Whipple observatory in southern Arizona $(1.3 \mathrm{~km}$ a.s.1., N $31^{\circ} 40^{\prime}$, W $110^{\circ} 57^{\prime}$ ). It consists of four Davies-Cotton telescopes (Davies \& Cotton 1957). Full array operations began in 2007 September. Each telescope has a focal length and a dish diameter of 12 meters and comprises 345 tessellated identical hexagonal mirror facets for a total effective mirror area of $106 \mathrm{~m}^{2}$. Cherenkov light from nearby extensive air showers is focused onto the cameras that consist of 499 photomultiplier tube (PMT) pixels. The spacing between the PMT pixels corresponds to 0.15 on the sky, resulting in a total camera field of view of 3.5. VERITAS has a large effective area (greater than $10^{5} \mathrm{~m}^{2}$ above $1 \mathrm{TeV}$ ), an energy resolution of $15 \%-20 \%$ and a single-event angular resolution of 0.08 (for energies greater than $500 \mathrm{GeV}$ ). This enables the detection of a point source flux that is $1 \%$ of the Crab nebula at a $5 \sigma$ significance in less than $30 \mathrm{hr}$ (Ong et al. 2009). For more details on the VERITAS instrument, see Holder et al. (2011).

VERITAS employs a three-level trigger system (Weinstein 2008). At the pixel level there must be a signal greater than $50 \mathrm{mV}$ (3-4 photoelectrons produced by $\sim 20$ photons), which is monitored by a constant fraction discriminator. An individual camera triggers when at least three adjacent pixels meet the first condition within a 5 ns time window. Finally, two or more telescopes must trigger within $50 \mathrm{~ns}$ for the event to be recorded. The photomultiplier tube relative gains are calculated using dedicated LED flasher calibration runs taken nightly (Hanna et al. 2010). Recorded showers are then parameterized by their second order moments, commonly referred to as Hillas parameters (Hillas 1985).

The data presented here were collected between 2009 and 2012. In order to reduce the systematic uncertainties in the background determination, observations were made using the wobble technique (Fomin et al. 1994; Berge et al. 2007). In 2009, all data were collected with the telescopes pointing to regions in the sky offset 0.5 from the HEGRA source position. In later seasons almost all the data from 2010 until 2012 were collected centering the telescopes on points equidistant from both TeV J2032+4130 and Cygnus X-3 ${ }^{33}$ (see Figure 1). A small amount of data was taken with the telescopes aimed at four wobble positions offset 0.5 from Cygnus X-3.

A total of $48.2 \mathrm{hr}$ of data was selected after removing data taken under bad weather conditions. TeV J2032+4130 was observed with a mean telescope elevation of $68^{\circ}$. The data were analyzed using the standard VERITAS calibration and reconstruction tools (Daniel 2008). Images from all participating telescopes in an event are combined to obtain the parameters of the arriving gamma-ray (Fegan 1997; Krawczynski et al. 2006). In order to suppress the large number of cosmic ray background events, gamma-ray/hadron separation criteria (cuts) are employed that compare the shapes of the shower images with those from simulated gamma-ray images. The results presented here required at least three telescopes to have recorded images of the shower with more than 1000 photons in each image. An additional cut on the square of the arrival angle of the incoming

\footnotetext{
33 Cygnus X-3 is 0.5 south of TeV J2032+4130.
}

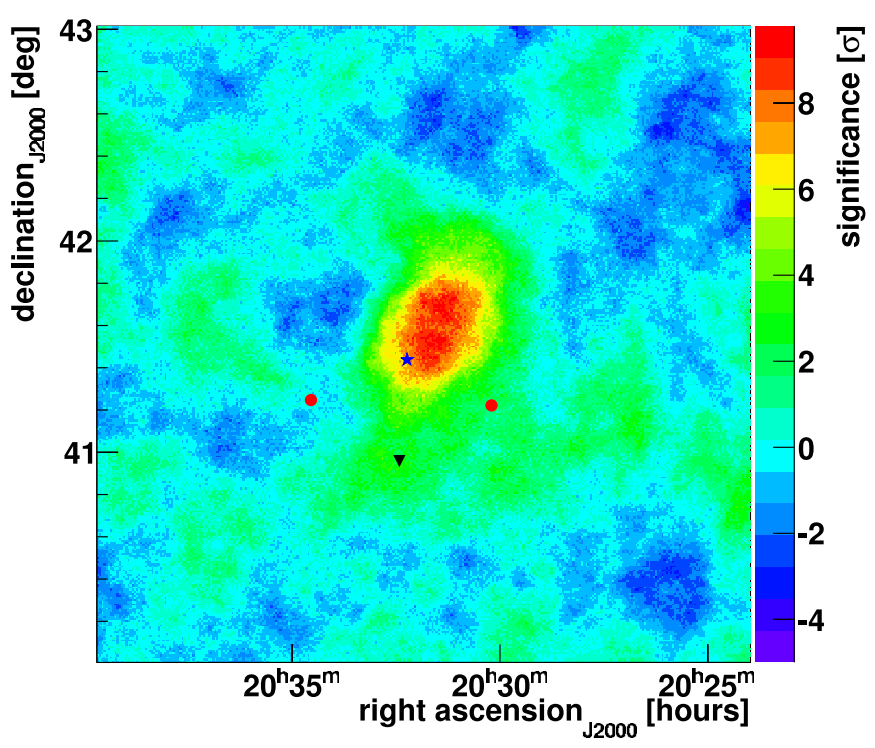

Figure 1. VERITAS gamma-ray significance map centered on VER J2031+415. The color indicates the significance within an integration window of 0.23 . The blue star marks the location of the Fermi-LAT pulsar PSR J2032+4127, the black triangle is the location of Cygnus X-3 and the red circles represent the shared wobble locations.

(A color version of this figure is available in the online journal.)

gamma ray with respect to the source position $\left(\theta^{2}<0.055\right)$ is applied to extract the signal. The ring background model has been used to estimate the background (Berge et al. 2007). The cuts have been previously optimized using a simulated source that has a flux $5 \%$ of the flux of the Crab nebula, and whose spectrum is at least as hard as the Crab nebula. The resulting analysis threshold is $520 \mathrm{GeV}$, which corresponds to the peak of the reconstructed energy distribution. A significance is calculated using the surviving gamma-ray like events and Equation (17) in Li \& Ma (1983).

\section{VERITAS RESULTS}

The analysis resulted in 595 events recorded in the source region and 3054 events in the selected background region. The geometrical background region selected was 7.7 times larger than the source region. This yields a significance of 8.7 standard deviation standard deviations at the HEGRA position of TeV J2032+4130. Figure 1 shows the VERITAS significance sky map. The gamma-ray point spread function for the analysis is 0.08 . The map is smoothed with a top-hat function corresponding to the source integration radius of 0.23 . The morphology of the source is investigated by binning the uncorrelated acceptance-corrected map of excess events. The uncorrelated excess map is fit to a two-dimensional Gaussian distribution in order to estimate the extent of the emission. The $\chi^{2}$ for the fit is 572.2 for 435 degrees of freedom. It is found to be asymmetric with an extension along the major axis of $9.5 \pm 1.2$, oriented to the northwest by $63^{\circ} \pm 6^{\circ}$, and $4^{\prime} .0 \pm 0.5$ along the minor axis, the quoted errors are statistical. The position of the centroid was found to be $20^{\mathrm{h}} 31^{\mathrm{m}} 40^{\mathrm{s}} \pm 65^{\mathrm{s}}$ and $41^{\circ} 33^{\prime} 53^{\prime \prime} \pm 37^{\prime \prime}$, which is consistent within errors with previous measurements, and we assign the name VER J2031+415.

The spectrum, shown in Figure 2, is well fit by a power law with a spectral index of $2.10 \pm 0.14_{\text {stat }} \pm 0.21_{\text {sys }}$ and normalization at $1 \mathrm{TeV}$ of $\left(9.5 \pm 1.6_{\text {stat }} \pm 2.2_{\text {sys }}\right) \times 10^{-13} \mathrm{TeV}^{-1} \mathrm{~cm}^{-2} \mathrm{~s}^{-1}$. The $\chi^{2}$ per degree of freedom is $4.5 / 5=0.9$. We found no 


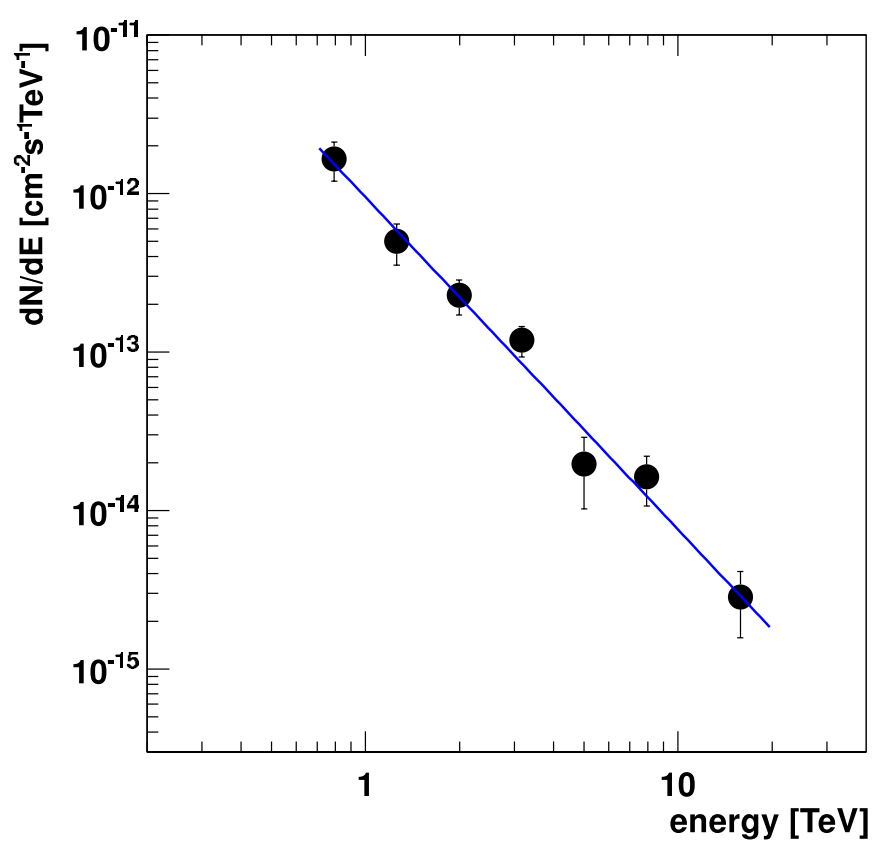

Figure 2. VERITAS differential gamma-ray spectrum of VER J2031+415. The blue line shows the power-law fit to the data points. A list of flux points can be found in Table 2.

(A color version of this figure is available in the online journal.)

Table 2

Differential Flux of VER J2031+415 Measured by VERITAS

\begin{tabular}{cccc}
\hline \hline $\begin{array}{l}\text { Energy } \\
(\mathrm{TeV})\end{array}$ & $\begin{array}{c}\text { Bin Width } \\
(\mathrm{TeV})\end{array}$ & $\begin{array}{c}\text { Flux } \\
\left(\mathrm{TeV}^{-1} \mathrm{~cm}^{-2} \mathrm{~s}^{-1}\right)\end{array}$ & $\begin{array}{c}\text { Significance } \\
(\sigma)\end{array}$ \\
\hline 0.79 & 0.37 & $(1.7 \pm 0.5) \times 10^{-12}$ & 4.0 \\
1.26 & 0.58 & $(5.0 \pm 1.5) \times 10^{-13}$ & 3.9 \\
2.00 & 0.93 & $(2.3 \pm 0.6) \times 10^{-13}$ & 4.8 \\
3.16 & 1.47 & $(1.2 \pm 0.3) \times 10^{-13}$ & 6.1 \\
5.01 & 2.33 & $(2.0 \pm 0.9) \times 10^{-14}$ & 2.5 \\
7.94 & 3.69 & $(1.6 \pm 0.6) \times 10^{-14}$ & 4.0 \\
15.85 & 15.12 & $(2.9 \pm 1.3) \times 10^{-15}$ & 2.8 \\
\hline
\end{tabular}

Notes. Also see Section 3 and Figure 2. The errors quoted are the $1 \sigma$ statistical errors.

evidence of a cut-off up to $20 \mathrm{TeV}$. The total integrated flux above $1 \mathrm{TeV}$ is $4.3 \%$ of the Crab nebula flux. This corresponds to a flux of $(2.35 \pm 0.55) \times 10^{-12} \mathrm{erg} \mathrm{cm}^{-2} \mathrm{~s}^{-1}$ above $1 \mathrm{TeV}$ and represents $0.3 \%$ of the spin-down luminosity of the pulsar PSR J2032+4127 (with an $\dot{E}$ of $2.7 \times 10^{35} \mathrm{erg} \mathrm{s}^{-1}$ ). Results of the spectral calculation can be seen in Table 2 .

Figure 3 shows the VERITAS significance sky maps for three different energy ranges: $E<1.46 \mathrm{TeV}, 1.46<E<2.7 \mathrm{TeV}$ and $E>2.7 \mathrm{TeV}$. The energy division was chosen a priori in an attempt to have equal statistics in each bin. Also shown is the one-dimensional histogram of the uncorrelated excess events along the major axis of the emission, as shown by the white boxes (which are $1.5 \times 0.3$ in extent). A Gaussian is fit to the excess. The dotted lined Gaussian is a set of Crab nebula data analyzed using the same analysis chain. This represents the response of the analysis to a point source. From this information the intrinsic width can be unfolded. The Crab Nebula is a strong source compared to TeV J2032+4130; a check has been made by reducing the Crab Nebula excess events by a factor of 20 and no significant change in the point source response was observed. The results can be seen in Table 3; based on the derived intrinsic width, no energy dependent morphology is observed.
Table 3

Gaussian Fits to Slices of the Uncorrelated Excess; see Figure 3

\begin{tabular}{|c|c|c|c|c|}
\hline $\begin{array}{l}\text { Energy } \\
\text { Range } \\
(\mathrm{TeV})\end{array}$ & $\begin{array}{l}\text { Point Source } \\
\text { Width } \\
\text { (deg) } \\
\text { Dotted Line }\end{array}$ & $\begin{array}{c}\text { Total } \\
\text { Width } \\
\text { (deg) } \\
\text { Solid Line }\end{array}$ & $\begin{array}{c}\text { Intrinsic } \\
\text { Width } \\
\text { (deg) }\end{array}$ & $\chi^{2} /$ Degrees of Freedom \\
\hline$\leqslant 1.46$ & 0.051 & $0.19 \pm 0.01$ & $0.18 \pm 0.03$ & $68.1 / 12$ \\
\hline $1.46-2.76$ & 0.046 & $0.33 \pm 0.05$ & $0.33 \pm 0.10$ & $12.4 / 12$ \\
\hline$\geqslant 2.76$ & 0.044 & $0.24 \pm 0.04$ & $0.24 \pm 0.09$ & $15.0 / 11$ \\
\hline
\end{tabular}

Notes. Widths are defined as 1 standard deviation. The $\chi^{2}$ per degree of freedom for the fit to the total width is shown in the final column. Note the error on the point source width is dominated by systematic errors.

Table 4

Upper Limits on Gamma-ray Emission from the Region of TeV J2032+4130 from the Analysis of Fermi-LAT Data

\begin{tabular}{lcc}
\hline $\begin{array}{l}\text { Energy Range } \\
(\mathrm{GeV})\end{array}$ & $\left.\begin{array}{c}99 \% \text { Upper Limit } \\
(\text { photons cm }\end{array} \mathrm{s}^{-1}\right)$ & $\begin{array}{c}99 \% \text { Upper Limit } \\
\left(\mathrm{erg} \mathrm{cm}^{-2} \mathrm{~s}^{-1}\right)\end{array}$ \\
\hline $0.5-1$ & $5.73 \times 10^{-9}$ & $1.18 \times 10^{-11}$ \\
$1-10$ & $1.94 \times 10^{-9}$ & $1.12 \times 10^{-12}$ \\
$10-100$ & $2.06 \times 10^{-11}$ & $1.20 \times 10^{-13}$ \\
\hline
\end{tabular}

\section{FERMI-LAT ANALYSIS}

An analysis of the Fermi-LAT data from the region around VER J2031+415 has been performed in the energy range $500 \mathrm{MeV}$ to $100 \mathrm{GeV}$. The Fermi-LAT is an electron-positron pair-conversion telescope (Atwood et al. 2009). The analysis described here uses data taken during the first four years of the operation of the Fermi-LAT detector. Data reduction was performed using the publicly available LAT data tools (version v9r27) and follows the analysis scheme described in Atwood et al. (2009). Initially, all events within a $20^{\circ}$ region of interest around VER J2031+415 were selected for further analysis. The resulting data set were analyzed using a binned likelihood technique (Cash 1979; Mattox et al. 1996). The routine is implemented in the LAT data tools as gtlike, which calculates the likelihood function probability using a source model folded with the LAT instrument response function ( $P 7 V 6)$. Finally a model of the source region was compared to the counts map to search for a signal.

The study of the associated diffuse source required us to take into account the pulsed emission of PSR J2032+4127 and assign phases to the gamma-ray photons and select only those in an off-pulse window, thereby minimizing contributions from the pulsar. Accurate timing solutions based on radio data from the Green Bank Telescope were used in conjunction with TEMPO2 (Hobbs et al. 2006) in order to phase fold the photon data.

For the timing analysis, photons between $100 \mathrm{MeV}$ and 300 $\mathrm{GeV}$ and within 0.5 of the pulsar position R.A. $=20^{\mathrm{h}} 32^{\mathrm{m}} 13.1$, decl. $=+41^{\circ} 27^{\prime} 24^{\prime \prime} .6$ were selected. Following the pulsar analysis, the off-pulse region was identified (approximately $20 \%$ of the total time was removed) and a binned likelihood analysis was again performed. No significant emission from the region of VER J2031+415 was observed and therefore we compute the $99 \%$ upper limits (Rolke et al. 2005). The assumed spectral index was 2.0 and the calculated results are shown in Table 4. 

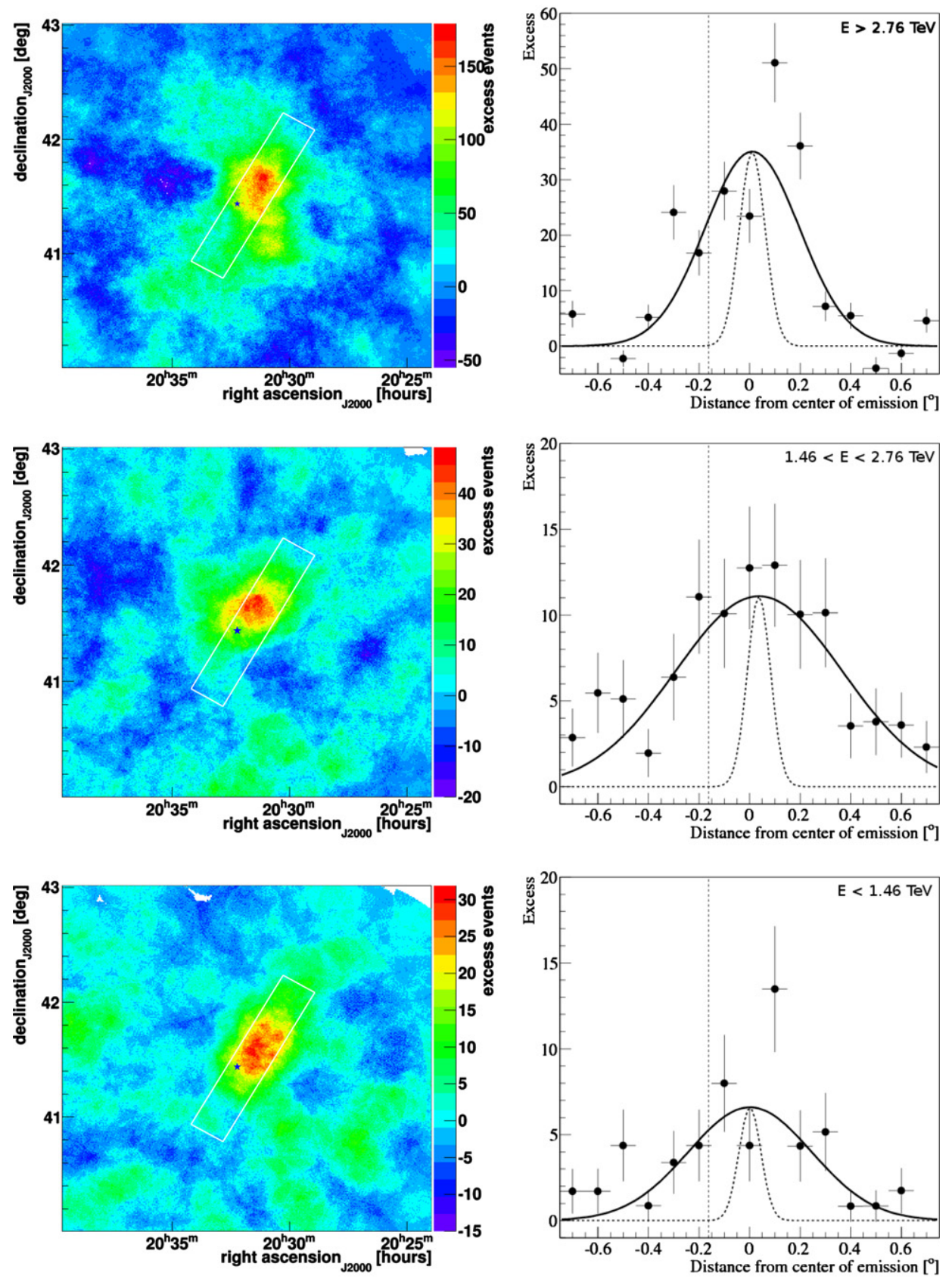

Figure 3. Left: correlated excess maps in the energy bands less than $1.46 \mathrm{TeV}, 1.46-2.72 \mathrm{TeV}$ and above $2.72 \mathrm{TeV}$. The white boxes represents a cross section aligned with the major axis of the excess. The Fermi-LAT pulsar PSR J2032+4127 is marked by the blue star. Right: slices of the uncorrelated excess map. The excess is fitted to a Gaussian distribution shown by the solid black line. Also plotted is the instrument response to a point source following the same analysis procedure (dotted line). The vertical dotted line represents the position of the Fermi-LAT pulsar. See the text for details.

(A color version of this figure is available in the online journal.)

\section{MULTIWAVELENGTH PROPERTIES AND INTERPRETATIONS}

Ever since its discovery by HEGRA (Aharonian et al. 2002), multiple observational efforts have been undertaken to identify the potential counterparts of $\mathrm{TeV} \mathrm{J} 2032+4130$ at other wavelengths. However, after years of multiwavelength observations, the origin of gamma-ray emission from the region still remains unresolved.

The Milagro water Cherenkov detector has performed a largescale survey of the Cygnus region and has discovered a population of extended sources without compelling counterparts (Abdo et al. 2007). Figure 4 shows the $8 \mu \mathrm{m}$ Midcourse Space
Experiment (MSX) map of the region with the Milagro and VERITAS $5 \sigma$ contours overlaid in black and white, respectively. The VERITAS flux has been integrated over a circular region with radius of 0.23 whereas, the Milagro flux has been derived over a region of $3^{\circ} \times 3^{\circ}$, owing to the larger angular resolution of the detector. It is of course quite possible that the flux of gamma rays measured by Milagro is not exclusively from $\mathrm{TeV}$ J2032+4130 but also contains a significant diffuse (or yet unresolved sources in the region) component. These observations are joined by those of the ground array detector ARGO (Bartoli et al. 2012). MAGIC and VERITAS measure spectral indices of $2.0 \pm 0.3_{\text {stat }}$ and $2.10 \pm 0.14_{\text {stat }}$, respectively. 


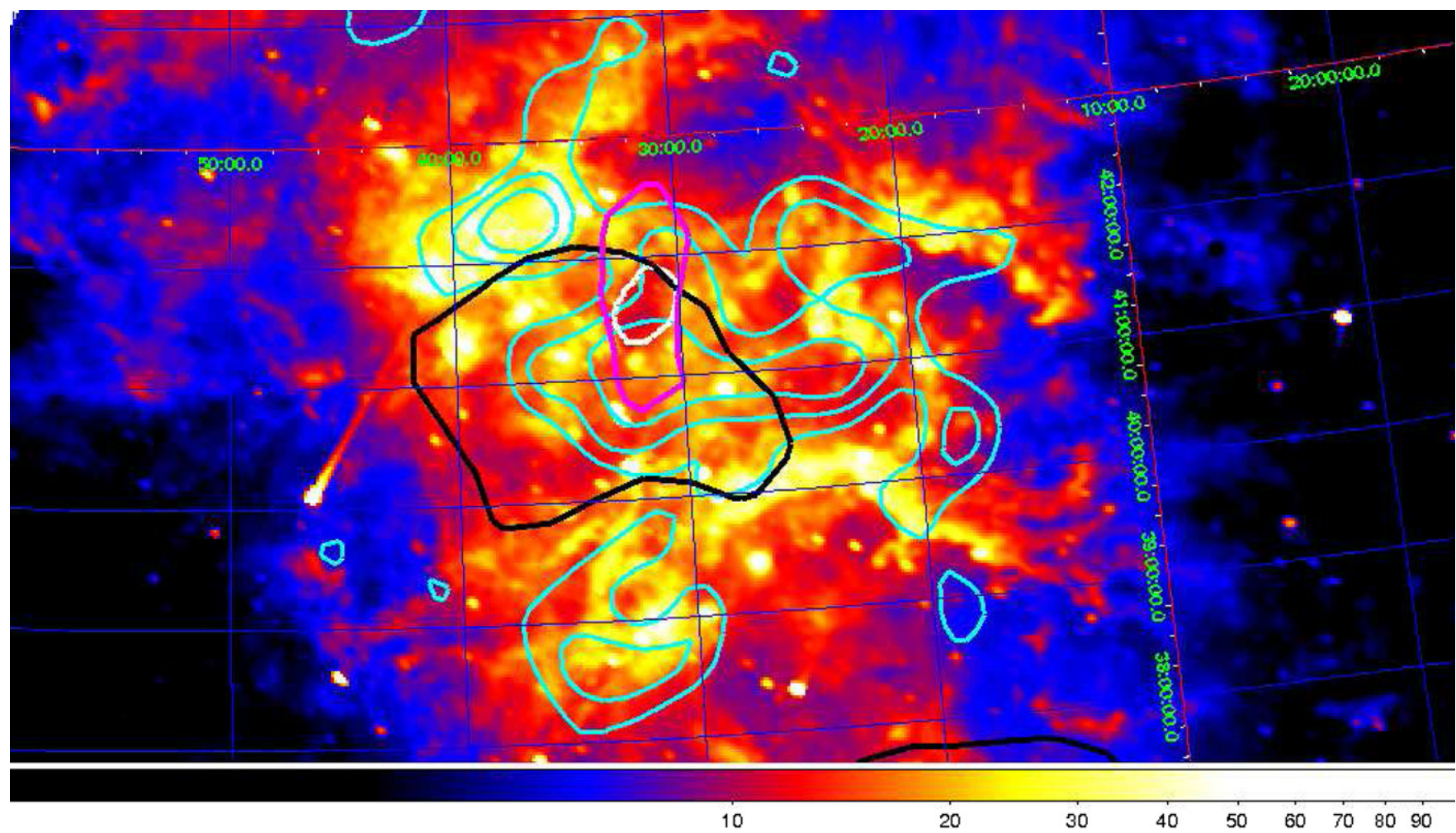

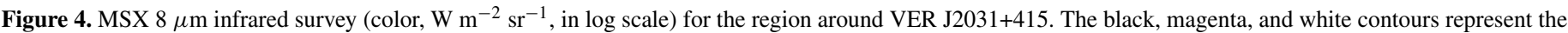
Milagro, ARGO, and VERITAS 5 standard deviation level. The Fermi-LAT 0.16, 0.24 and 0.32 photons bin ${ }^{-1}$ contours are shown in cyan.

(A color version of this figure is available in the online journal.)

In comparison both the Milagro and ARGO observations resulted in significantly softer spectral indices of $3.2 \pm 0.2$ and $2.8 \pm 0.4$. ARGO operates at comparable energies to those of the imaging Cherenkov telescopes and sees an extension that is twice as large.

At $\mathrm{GeV}$ gamma-ray energies Fermi-LAT has reported the existence of a cocoon located in the Cygnus region, spread over $2^{\circ}$ between VER J2031+415 and $\gamma$-Cygni. Even though pulsar wind nebulae (PWNe) are known to power extended gamma-ray sources, it is unlikely that the cocoon is powered by PSR J2032+4127 due to its size. Ackermann et al. (2011) suggest that the gamma-ray excess of the cocoon is due to a population of freshly accelerated cosmic rays. The link between the cocoon and the emission at $\mathrm{TeV}$ energies remains unclear and we cannot rule out any connection between the two.

Figure 5 shows multiwavelength images of VER J2031+415 and its vicinity. Assuming a distance of $1.7 \mathrm{kpc}$, the one $\sigma$ width of the $\mathrm{TeV}$ gamma-ray emission is $4.7 \times 2.0 \mathrm{pc}$ along the major and minor axes. Due to the star-forming activity known to take place in Cygnus X, one of the richest known regions of star formation in the Galaxy, the infrared (IR) and radio images are dominated by bright diffuse emission exhibiting complex and intricate structure. Interestingly, nearly all the $\mathrm{TeV}$ gamma-ray emission happens to be confined within one of the rare voids. Although a chance coincidence is possible, the rarity of these voids and the similarity between the $\mathrm{TeV}$ source morphology and that of the void hint at a possible physical connection between the two. The large size of the void and lack of a characteristic patch structure make it dissimilar to the dark infrared clouds (composed of cold molecular material) that were recently found within the Cygnus X complex (Hora et al. 2009). Moreover, CO line imaging (see Butt et al. 2003) shows that compact $\mathrm{CO}$ emission is only seen in the eastern part of the void and of the extended $\mathrm{TeV}$ source. Hence, it does not seem likely that the void is due to absorption by cold molecular material along our line-of-sight.

One possibility is that the void is formed due to the collective action of powerful stellar winds from an association of massive stars, a hypothesis considered by Butt et al. (2003) and Albert et al. (2008). They argued that the presence of a large, mechanical power density from the stellar winds of the OB stars, make Cygnus OB2 a prime candidate for the investigation of the stellar wind hypothesis for the acceleration of Galactic cosmic rays. Massive stars are common in Cygnus OB2 and the energy required to power the VHE emission is only a fraction of the estimated wind kinetic energy. However, many of these massive stars are outside the observed $\mathrm{TeV}$ gamma-ray emission region (see cyan circles in Figure 5, top panels). Thus the observed $\mathrm{TeV}$ gamma-ray morphology seems unlikely to be produced by massive stellar winds.

It is possible that the void is due to a supernova (SN) explosion in Cygnus OB2 resulting in a SNR that expanded into the surrounding medium. Butt et al. (2008) mention hints of a shell-like structure with a radius of $\sim 5^{\prime}$ seen in the $6 \mathrm{~cm}$ Very Large Array (VLA) image. However, we note that this size is smaller than the size of the void and the $\mathrm{TeV}$ gamma-ray source extent. Although some faint non-thermal radio emission is present within VER J2031+415 (see Figure 6), it may be due to a PWN within the SNR rather than the SNR shell.

If the SN explosion occurred relatively long ago ( $\gtrsim 30,000 \mathrm{yr}$ ), the shell could have grown much larger and become fainter. In this scenario the $\mathrm{TeV}$ gamma-ray emission would come from the interior of the aged SNR filled with a pulsar wind. It would then be natural to assume that the SNR is linked to LAT 

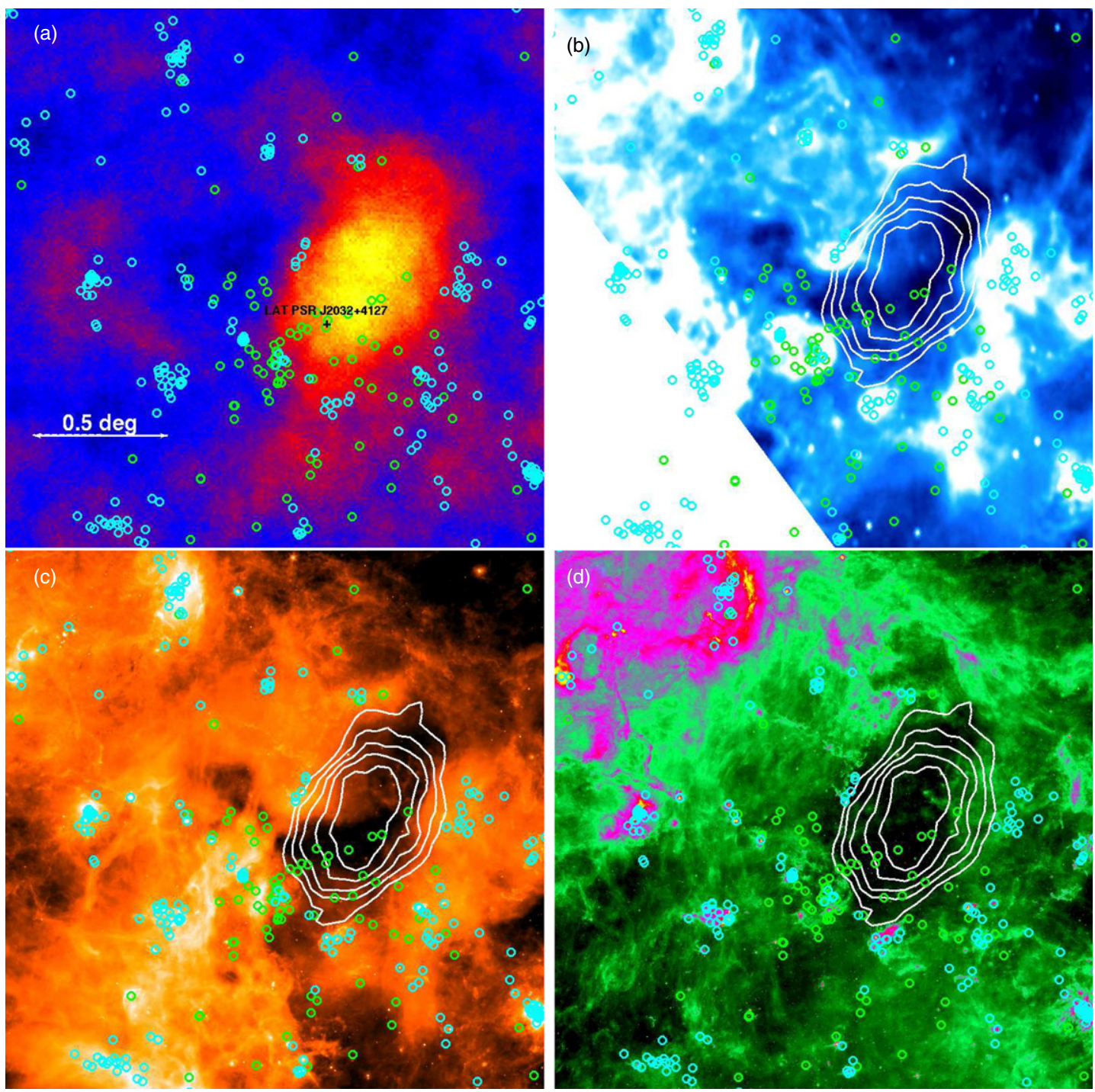

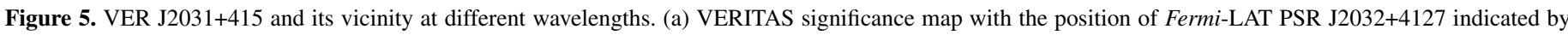

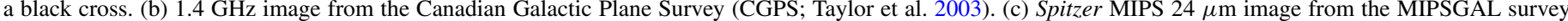

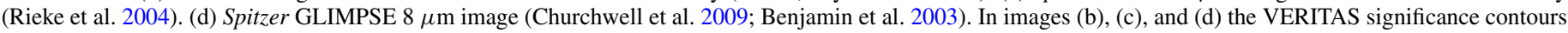

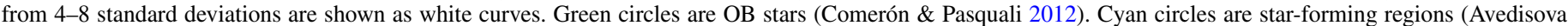
2002)

(A color version of this figure is available in the online journal.)

PSR J2032+4127, which is apparently accompanied by a faint X-ray PWN. The faintness of the X-ray PWN could be attributed to the large spin-down age of the pulsar and comparatively low $\dot{E}$. Based on a distance of $1.7 \mathrm{kpc}$ the gamma-ray efficiency is $\epsilon_{\gamma}=L_{\gamma} / \dot{E}=0.3 \%$ (in the energy range $1-10 \mathrm{TeV}$ ), while the $\mathrm{X}$-ray PWN efficiency is only $0.04 \%$ (between 0.5 and $8.0 \mathrm{keV}$ ).

The position of the pulsar suggests that it might be moving South-East along the elongation of the TeV gamma-ray emission. Given the observed angular separation between the center of emission and the pulsar, the transverse velocity is calculated to be $51 \mathrm{~km} \mathrm{~s}^{-1}$, assuming a characteristic age of $0.11 \mathrm{Myr}$ and a distance of $1.7 \mathrm{kpc}$.

The majority of identified Galactic TeV gamma-ray sources are PWNe (Kargaltsev et al. (2012). Adopting the PWN scenario for VER J2031+415 with PSR J2023+4157 as the pulsar powering the TeV PWN, one can compare this source to other $\mathrm{X}$-ray/TeV PWNe and PWNe candidates. PSR J2032+4127 is one of the oldest and weakest pulsars (in terms of $\dot{E}$ ) whose
PWN is detected both in X-ray and TeV gamma-rays, (see Figure 7), Geminga being the other notable example. The TeV gamma-ray spectrum of VER J2031+415 is fit by a powerlaw with spectral index $\simeq 2$, one of the hardest among PWNe and PWNe candidates. Because of this hard index, the spectrum must exhibit a cut-off not too far from $10 \mathrm{TeV}$ due to the Klein-Nishina effect in order to be consistent with the PWN interpretation. The X-ray luminosity of the PWN (at $d=1.7 \mathrm{kpc}$ ) is unremarkable (see Figure 7) and consistent with the spin-down properties of the pulsar. Finally, the distance-independent ratio of the $\mathrm{TeV}$ to $\mathrm{X}$-ray luminosity for VER J2031+415 is fairly well constrained and consistent with the expectations.

Interestingly, there is another $\mathrm{TeV}$ gamma-ray source (HESS J1646-458; Abramowski et al. (2012); potentially a PWN of PSR J1648-4611) whose spin-down properties are not too different from those of PSR J2032+4127 apparently also located within the molecular cavity in the general direction of 


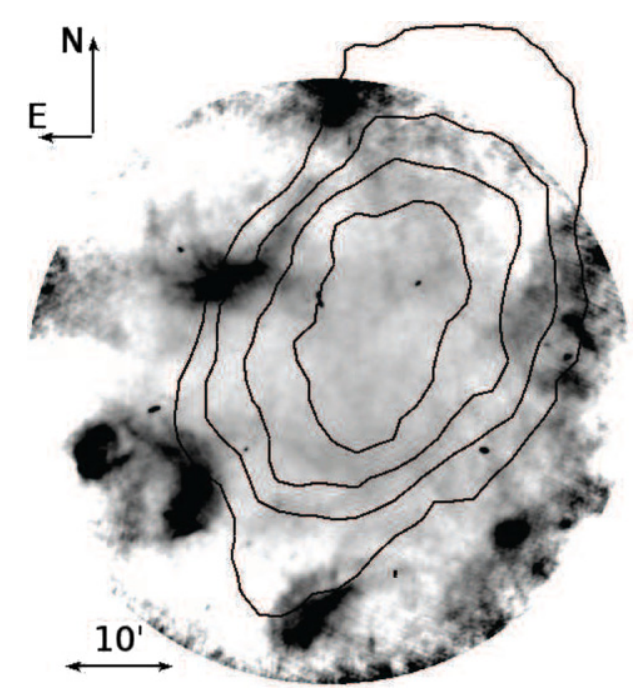

Figure 6. VLA $20 \mathrm{~cm}$ ( $L$-band) image with VERITAS $4 \sigma$ to $8 \sigma$ contours overlaid (Condon et al. 1998)
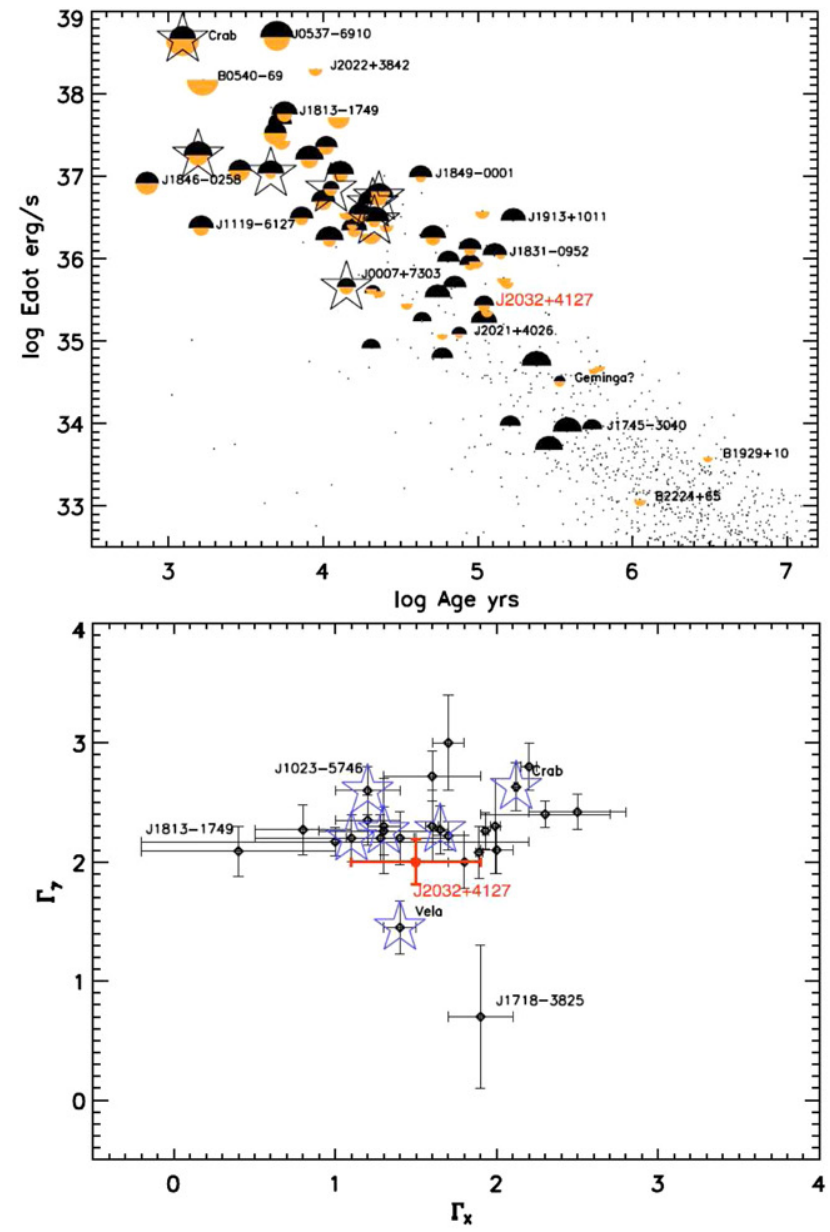

Westerlund 1 (Luna et al. 2010). However, the X-ray PWN of PSR J1648-4611 has not yet been detected and its TeV PWN classification still remains to be proved.

\section{CONCLUSIONS}

VERITAS has made a deep observation of TeV J2032+4130 resulting in a significance of 8.7 standard deviation which is currently the most sensitive measurement made on this source in gamma rays from 0.5 to $20 \mathrm{TeV}$. The position and the measured energy spectrum are found to be consistent with previous measurements made by other IACTs. The latter is fit well by a power-law with no evidence of a cutoff. The centroid of the emission has been measured to a greater accuracy than before, and for the first time an intrinsic asymmetry in the morphology has been found. Almost all the TeV gamma-ray emission has been found to come from a region that is seen as a void in both radio and infrared wavebands. After considering multiwavelength data, we favor a relic PWN scenario for TeV J2032+4130 powered by the pulsar PSR J2032+4157. However, the possibility that the $\mathrm{TeV}$ gamma rays are produced by stellar
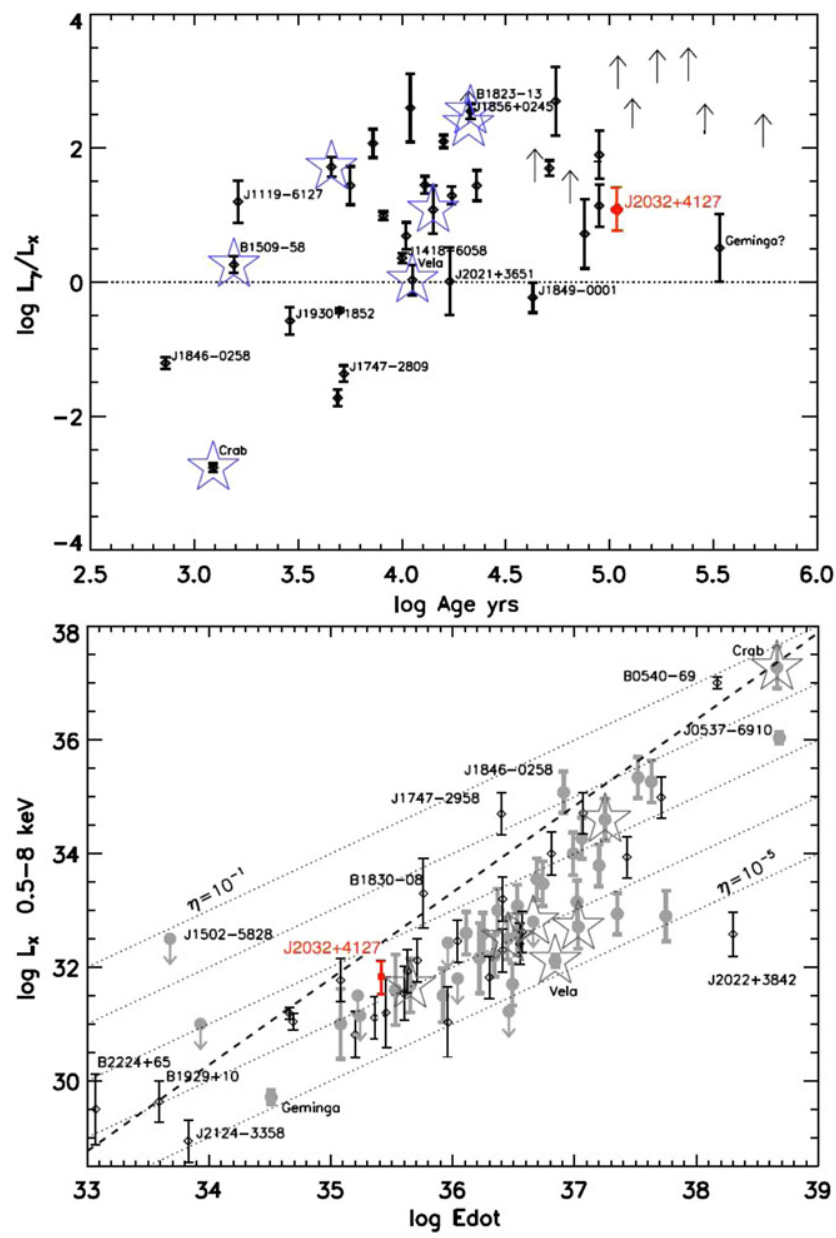

Figure 7. Top left: pulsars with detected PWNe (or PWN candidates) in the $\tau_{\mathrm{sd}}-\dot{E}$ diagram. The semi-circles correspond to X-ray (orange) and TeV (black) PWNe, their sizes are proportional to logarithms of the corresponding PWN luminosities. The small black dots denote the pulsars from the ATNF catalog Manchester et al. (2005). Pulsars with PWNe detected by Fermi are marked by stars. Top right: TeV-to-X-ray luminosity ratio vs. pulsar's age for PWNe and PWN candidates. Limits are shown by arrows. The PWNe detected by Fermi are marked by stars. Uncertain detections are shown by thin lines. The dotted lines corresponds to $L_{\gamma}=L_{X}$. Bottom left: TeV vs. X-ray photon indices for PWNe and PWN candidates. Bottom right: X-ray luminosities of PWNe and PWN candidates vs. pulsar's $\dot{E}$. TeV PWNe and TeV PWN candidates are shown with gray thick error bars. The dotted straight lines correspond to constant X-ray efficiencies; the upper bound, $\log L_{X}^{\mathrm{cr}}=1.51 \log \dot{E}-21.4$ is shown by a dashed line. The PWNe detected in GeV by Fermi are marked by stars. Figures adapted from Kargaltsev et al. (2013). In all panels PWN of PSR $\mathrm{J} 2032+4127$ is marked in red.

(A color version of this figure is available in the online journal.) 
winds cannot be ruled out, despite the relatively fewer number of massive stars in the void.

This research is supported by grants from the U.S. Department of Energy Office of Science, the U.S. National Science Foundation and the Smithsonian Institution, by NSERC in Canada, by Science Foundation Ireland (SFI 10/RFP/AST2748) and by STFC in the U.K. We acknowledge the excellent work of the technical support staff at the Fred Lawrence Whipple Observatory and at the collaborating institutions in the construction and operation of the instrument. G.H. acknowledges support through the Young Investigators Program of the Helmholtz Association. The work by O.K. was supported by NASA grants NNX09AC84G and NNX09AC81G.

\section{REFERENCES}

Abdo, A. A., Abeysekara, U., Allen, B. T., et al. 2012, ApJ, 753, 159 Abdo, A. A., Allen, B., Berley, D., et al. 2007, ApJL, 658, L33

Abramowski, A., Acero, F., Aharonian, F., et al. 2012, A\&A, 537, A114 Ackermann, M., Ajello, M., Allafort, A., et al. 2011, Sci, 334, 1103 Aharonian, F., Akhperjanian, A., Beilicke, M., et al. 2002, A\&A, 393, L37 Aharonian, F., Akhperjanian, A., Beilicke, M., et al. 2005, A\&A, 431, 197 Albert, J., Aliu, E., Anderhub, H., et al. 2008, ApJL, 675, L25 Atwood, W. B., Abdo, A. A., Ackermann, M., et al. 2009, ApJ, 697, 1071 Avedisova, V. S. 2002, yCat, 5112, 0

Bartoli, B., Bernardini, P., Bi, X. J., et al. 2012, ApJL, 745, L22

Benjamin, R. A., Churchwell, E., Babler, B. L., et al. 2003, PASP, 115, 953

Berge, D., Funk, S., \& Hinton, J. 2007, A\&A, 466, 1219

Butt, Y. M., Benaglia, P., Combi, J. A., et al. 2003, ApJ, 597, 494

Butt, Y. M., Combi, J. A., Drake, J., et al. 2008, MNRAS, 385, 1764

Butt, Y. M., Drake, J., Benaglia, P., et al. 2006, ApJ, 643, 238

Camilo, F., Ray, P. S., Ransom, S. M., et al. 2009, ApJ, 705, 1

Cash, W. 1979, ApJ, 228, 939

Churchwell, E., Babler, B. L., Meade, M. R., et al. 2009, PASP, 121, 213

Comerón, F., \& Pasquali, A. 2012, A\&A, 543, A101

Condon, J. J., Cotton, W. D., Greisen, E. W., et al. 1998, AJ, 115, 1693

Cordes, J. M., \& Lazio, T. J. W. 2002, arXiv:astro-ph/0207156

Cui, W. 2009, RAA, 9, 841
Daniel, M. K. 2008, in International Cosmic Ray Conference, Vol. 3 ed. R. Caballero, J. C. D’Olivo, G. Medina-Tanco, L. Nellen, F. A. Sánchez, \& J. F. Valdés-Galicia, 1325

Davies, J. M., \& Cotton, E. S. 1957, SOE, 1, 16

Fegan, D. J. 1997, JPhG, 23, 1013

Fomin, V. P., Stepanian, A. A., Lamb, R. C., et al. 1994, APh, 2, 137

Hanna, D., McCann, A., McCutcheon, M., \& Nikkinen, L. 2010, NIMPA, 612,278

Hillas, A. M. 1985, in International Cosmic Ray Conference, Vol. 3, ed. F. C. Jones, J. Adams, \& G. M. Mason (Hanover, MD: NASA STI), 445

Hobbs, G. B., Edwards, R. T., \& Manchester, R. N. 2006, MNRAS, 369, 655

Holder, J., Aliu, E., Arlen, T., et al. 2011, in Proc. 32nd ICRC, Beijing (arXiv:1111.1225)

Hora, J. L., Bontemps, S., Megeath, S. T., et al. 2009, BAAS, 41, 498

Horns, D., Hoffmann, A. I. D., Santangelo, A., Aharonian, F. A., \& Rowell, G. P. 2007, A\&A, 469, L17

Kargaltsev, O., Pavlov, G. G., \& Durant, M. 2012, in ASP Conf. Ser. 466, Electromagnetic Radiation from Pulsars and Magnetars, ed. W. Lewandowski, O. Maron, \& J. Kijak (San Francisco, CA: ASP), 167

Kargaltsev, O., Rangelov, B., \& Pavlov, G. G. 2013, in The Universe Evolution: Astrophysical and Nuclear Aspects, ed. I. Strakovsky \& L. Blokhintsev (San Francisco, CA: ASP), 466

Konopelko, A., Atkins, R. W., Blaylock, G., et al. 2007, ApJ, 658, 1062

Krawczynski, H., Carter-Lewis, D. A., Duke, C., et al. 2006, APh, 25, 380

Lang, M. J., Carter-Lewis, D. A., Fegan, D. J., et al. 2004, A\&A, 423, 415

Li, T.-P., \& Ma, Y.-Q. 1983, ApJ, 272, 317

Luna, A., Mayya, Y. D., Carrasco, L., \& Bronfman, L. 2010, ApJL, 713, L45

Manchester, R. N., Hobbs, G. B., Teoh, A., \& Hobbs, M. 2005, AJ, 129, 1993

Mattox, J. R., Bertsch, D. L., Chiang, J., et al. 1996, ApJ, 461, 396

Mukherjee, R., Halpern, J. P., Gotthelf, E. V., Eracleous, M., \& Mirabal, N. 2003, ApJ, 589, 487

Murakami, H., Kitamoto, S., Kawachi, A., \& Nakamori, T. 2011, PASJ, 63, 873

Ong, R. A., Acciari, V. A., Arlen, T., et al. 2009, arXiv:0912.5355

Paredes, J. M., Martí, J., Ishwara Chandra, C. H., \& Bosch-Ramon, V. 2007, ApJL, 654, L135

Rieke, G. H., Young, E. T., Engelbracht, C. W., et al. 2004, ApJS, 154, 25

Rolke, W. A., López, A. M., \& Conrad, J. 2005, NIMPA, 551, 493

Taylor, A. R., Gibson, S. J., Peracaula, M., et al. 2003, AJ, 125, 3145

The ARGO-YBJ Collaboration, Bartoli, B., Bernardini, P., et al. 2013, ApJ, 779,27

Weinstein, A. 2008, in International Cosmic Ray Conference, Vol. 3, ed. R. Caballero, J. C. D’Olivo, G. Medina-Tanco, L. Nellen, F. A. Sánchez, \& J. F. Valdés-Galicia, 1539 